\title{
Simulation of induction at low magnetic Prandtl number
}

\author{
Yannick PONTY and Hélène POLITANO \\ CNRS, UMR 6529, Observatoire de la Côte d'Azur BP 4229, Nice Cedex 4, France \\ Jean-François PINTON \\ CNRS, UMR 5672, Laboratoire de Physique, École Normale Supérieure, 46 allée d'Italie 69007 Lyon, France
}

(Dated: November 26, 2003)

\begin{abstract}
We consider the induction of magnetic field in flows of electrically conducting fluid at low magnetic Prandtl number and large kinetic Reynolds number. Using the separation between the magnetic and kinetic diffusive lengthscales, we propose a new numerical approach. The coupled magnetic and fluid equations are solved using a mixed scheme, where the magnetic field fluctuations are fully resolved and the velocity fluctuations at small scale are modelled using a Large Eddy Simulation (LES) scheme. We study the response of a forced Taylor-Green flow to an externally applied field: tology of the mean induction and time fluctuations at fixed locations. The results are in remarkable agreement with existing experimental data; a global $1 / f$ behavior at long times is also evidenced.
\end{abstract}

PACS numbers: $47.27 . \mathrm{Eq}, 47.65+\mathrm{a}, 52.65 \mathrm{Kj}, 91.25 \mathrm{Cw}$

One of the strongest motivation in the study of nonlinear effects in magnetohydrodynamics is that electrically conductive flows are capable of dynamo action: the stretching of magnetic field lines by the flow velocity gradients can exceed the (Joule) diffusion. A bifurcation threshold occurs, above which the self-generation of a magnetic field takes place. It has been validated in constrained flows of liquid sodium which mimic analytical models: the Karlsruhe [1] and Riga experiments [2]. The self-generation of a magnetic field in non-constrained homogeneous flows is still an open problem actively studied by many groups 3 . In this research, numerical studies have long played an important role. Kinematic dynamo simulations assume a given pattern of a stationary velocity field and study the initial linear growth rate of magnetic field perturbations. They have been extensively used to test the dynamo capacity of flow geometries and proved to be successful at determining the dynamo threshold in the Karlsruhe and Riga experiments [4, 5]. They have also shown that dynamo action is a possibility in unconstrained homogeneous flows of the von Kármán type [6, 7]. Another numerical approach is to perform Direct Numerical Simulations (DNS) of the full governing equations: the induction equation coupled with the fluid dynamical one by the Lorentz force, the flow being sustained by a given force (or equivalently an average geometry). They have confirmed that dynamo action is present in flows with differential rotations and helicity [8, 9, 10]. However, DNS are at present restricted to situations where the magnetic Prandtl number, $P_{m}=\nu / \lambda$ (where $\lambda$ is the magnetic diffusivity) is of order one, i.e. to situations where the smallest scales of the magnetic and velocity fields have the same characteristic size 11]. This is not the case in liquid metals, which have very small magnetic Prandtl number values e.g. $P_{m} \sim 10^{-6}$ for liquid Gallium and $P_{m} \sim 10^{-5}$ for liquid Sodium. Recall that, below the dynamo threshold, a stationary forced flow with a power input $\epsilon$ (in Watts per $\mathrm{kg}$ ) has a viscous dissipative scale $\eta_{u} \sim\left(\nu^{3} / \epsilon\right)^{1 / 4}$ and a Joule diffusive scale $\eta_{B} \sim\left(\lambda^{3} / \epsilon\right)^{1 / 4}$ - hence a ratio $\eta_{u} / \eta_{B} \sim P_{m}^{3 / 4}$. Therefore, at low $P_{m}$, the magnetic diffusive length scale is very much larger than the velocity dissipative scale. If non-linear effects are to develop, the magnetic Reynolds number $R_{m} \sim U L / \lambda$ (where $U$ and $L$ represent the characteristic velocity and scale of the flow) must be at least of order one and thus the kinetic Reynolds number of the flow, Re $\sim U L / \nu \sim R_{m} / P_{m}$, must be very large (turbulence is fully developed). A DNS treatment of such a system is at present out of reach.

In this paper, we present a new approach for the study of the magnetic induction in large $R e$ - low $P_{m}$ flows; we restrict ourselves to regimes below the dynamo threshold. In this parameter region, the magnetic field "lives" essentially within the large and inertial hydrodynamic scales. We thus propose to treat with a sub-grid model the velocity scales which are smaller than the magnetic diffusive length. Schemes using hyperviscosity have previously been used [4, 12]. Here, we prefer a LES approach, which has proved very successful for the simulation of turbulent flows with large scale structures and for the modelling of energy transfers [13]. In this hybrid scheme, we solve the induction equation on a fully resolved grid and we use a LES method for the velocity field, with a cut-off scale at the end of the magnetic diffusive range. We consider the response of a conductive fluid to an uniform magnetic field: topology of the mean induced field and spatio-temporal features of the magnetic fluctuations are studied. The chosen flow is a forced Taylor-Green vortex (TG). It shares many similarities with the experimental von Kármán swirling flows which have already been investigated in DNS near $P_{m} \sim \mathcal{O}(1)$ [9, 10].

In non-dimensional form, the incompressible MHD equations have two classical control parameters, the magnetic and kinetic Reynolds numbers, and one has to 
choose a forcing mechanism that generates the desired values of $R_{m}$ and $R e$. In order to be closer to experimental procedures, we prefer to fix the driving force and the magnetic Prandtl number. Hence, the dynamical time $t_{0}$ is set to the magnetic diffusion time scale, i.e. $t_{0} \lambda / L^{2} \sim \mathcal{O}(1)$, where $L$ is a length scale characteristic of the system size. Changes in magnetic diffusivity for real fluids would change that time scale. We write the MHD equations, with constant unit density, as

$$
\begin{aligned}
\partial_{t} \mathbf{u}+\mathbf{u} . \nabla \mathbf{u} & =-\nabla P+P_{m} \nabla^{2} \mathbf{u}+\mathbf{F}+(\nabla \times \mathbf{b}) \times \mathbf{B} 1 \\
\partial_{t} \mathbf{b} & =\nabla \times(\mathbf{u} \times \mathbf{B})+\nabla^{2} \mathbf{b} \\
\nabla \cdot \mathbf{u} & =0, \quad \nabla \cdot \mathbf{b}=0
\end{aligned}
$$

where $\mathbf{u}$ is the velocity field, $\mathbf{B}=\mathbf{B}_{0}+\mathbf{b}$ is the net magnetic field in the flow, sum of the applied and induced fields. Once the amplitude $F$ of the driving force is fixed, the (non-dimensional) rms intensity of the velocity fluctuations is $u_{r m s} \sim \sqrt{F}$, the Reynolds number is $R e \sim \sqrt{F} / P_{m}$ and the magnetic Reynolds number is $R_{m} \sim \sqrt{F}$. When the interaction parameter, ratio of the Lorentz force to the inertial forces, defined as $N \simeq B_{0}^{2} / u_{r m s} \sim B_{0}^{2} / \sqrt{F}$ is small, the back reaction of the induced field on the velocity field is negligeable. The above expressions are only dimensional estimates; in practice, the characteristic flow quantities are computed as mean temporal values from the data - cf. Table 1.

We use a parallelized pseudo-spectral code in a $[0-2 \pi]^{3}$ periodic box. Time stepping is done with an exponential forward Euler-Adams-Bashford scheme. The LES model is of the Chollet-Lesieur type [13] in which the kinematic viscosity $\nu$ is replaced in spectral space by an eddy viscosity. In Eq. (1) the magnetic Prandtl number is then replaced by:

$$
P_{m}(k, t)=0.1\left(1+5\left(k / K_{c}\right)^{8}\right) \sqrt{E_{v}\left(k=K_{c}, t\right) / K_{c}} .
$$

Here $K_{c}$ is the cut-off wavenumber of the velocity field, and $E_{v}(k, t)$ is the one-dimensional kinetic energy spectrum. The effective Prandtl number $P_{m_{\text {eff }}}$ is obtained as the temporal mean of $P_{m}(0, t)$. Note that the effective fluid viscosity $\nu_{\text {eff }}$ is of the same magnitude. A consistency condition for our approach is that the magnetic field fluctuations are fully resolved when $2 \pi / K_{c}$ is smaller than the magnetic diffusive scale $\eta_{B} \sim l_{0} / R_{m}^{3 / 4}, l_{0}$ being the integral scale computed from the kinetic energy spectrum. The flow is driven by the TG vortex geometry

$$
\mathbf{F}_{\mathrm{TG}}\left(k_{0}\right)=2 F\left[\begin{array}{c}
\sin \left(k_{0} x\right) \cos \left(k_{0} y\right) \cos \left(k_{0} z\right) \\
-\cos \left(k_{0} x\right) \sin \left(k_{0} y\right) \cos \left(k_{0} z\right) \\
0
\end{array}\right]
$$

$\left(k_{0}, k_{0}, k_{0}\right)$ is the wavevector that prescribes the velocity large scale (hereafter $k_{0}=1$ ). The $\mathbf{F}_{\mathrm{TG}}$ and $\mathbf{B}_{\mathbf{0}}$ amplitudes are chosen such that the interaction parameter $\mathrm{N}$ remains smaller than $10^{-2}$. After an initial transient $(t<10)$ the flow has reached a steady state: the kinetic energy fluctuates less than $3.5 \%$ around its mean value. All quantities are tracked up to $t_{\max }=410 t_{0}$ - note that $200 t_{0}$ is of the order of the measurement time in most Sodium experiments [14, 15, 16]. For comparison, the eddy turnover time $\tau_{N L} \sim l_{0} / u_{r m s}$ is given in Table 1 .

\begin{tabular}{|c|c|c|}
\hline RUN & $\# \mathbf{1} \mathbf{B}_{\mathbf{0}}=0.1 \hat{\mathbf{x}}$ & $\mathbf{\# 2} \mathbf{B}_{\mathbf{0}}=0.1 \hat{\mathbf{z}}$ \\
\hline$T G$ & $R e=9209$ & $R e=9212$ \\
$k_{0}=1$ & $R_{m}=6.65$ & $R_{m}=6.68$ \\
$F=3 / 2$ & $R_{l_{T}}=95.94$ & $R_{l_{T}}=95.96$ \\
$128^{3}$ grid points & $P_{m_{\text {eff }}} \sim 7.2210^{-4}$ & $P_{m_{\text {eff }}}=7.2610^{-4}$ \\
$K_{c}=k_{\max }-3$ & $N=8.2310^{-3}$ & $N=8.1810^{-3}$ \\
$k_{\max }=64$ & $l_{0}=2.338$ & $l_{0}=2.337$ \\
$t_{\max }=410$ & $l_{T}=0.024$ & $l_{T}=0.024$ \\
& $\eta_{B}=0.565$ & $\eta_{B}=0.563$ \\
& $\tau_{N L}=1.217$ & $\tau_{N L}=1.224$ \\
& $u_{r m s}=2.843$ & $u_{r m s}=2.858$ \\
& $b_{r m s}=0.061$ & $b_{r m s}=0.064$ \\
& $\max |\mathbf{u}|=8.211$ & $\max |\mathbf{u}|=8.249$ \\
& $\max |\mathbf{b}|=0.160$ & $\max |\mathbf{b}|=0.180$ \\
\hline
\end{tabular}

TABLE I: Time averaged quantities: $u_{r m s}=\left\langle\mathbf{u}^{2}\right\rangle^{1 / 2}, b_{r m s}=$ $\left\langle\mathbf{b}^{2}\right\rangle^{1 / 2}$, flow integral scale $l_{0}=2 \pi \sum_{k} E_{v}(k) / k / \sum_{k} E_{v}(k)$, Taylor microscale $l_{T} \sim l_{0} R_{e}^{-1 / 2}$, diffusive scale $\eta_{B}$ and eddy turnover time $\tau_{N L}$. Non-dimensional parameters: effective Prandtl number $P_{m_{\text {eff }}}$, kinetic Reynolds number $R_{e}=$ $l_{0} u_{r m s} / \nu_{\text {eff }}$ (see text), and magnetic Reynolds number $R_{m}=$ $P_{m_{\text {eff }}} R_{e}$, Taylo-based Reynolds number $R_{l_{T}} \sim R_{e}^{1 / 2}$, interaction parameter $N=R_{m} B_{0}^{2} / u_{r m s}^{2}$.

Figure 1 shows the power spectra of the velocity and magnetic field fluctuations with $\mathbf{B}_{\mathbf{0}}$ applied along the $\hat{\mathbf{x}}$-axis (a direction perpendicular to the rotation axis of the counter-rotating eddies of the TG cells). The kinetic energy spectrum exhibits the $k^{-5 / 3}$ Kolmogorov scaling law maintained throughout the range by the LES scheme. The peak at low wavenumber is due the large scale TG forcing, also visible on the magnetic field spectrum. The magnetic inertial range is well fitted by a $k^{-11 / 3}$ power law in agreement with a Kolmogorov phenomenology 17, 18]. The magnetic diffusive scale is reached within the computational box. The main goal of our numerical strategy is thus achieved: the magnetic fluctuations are fully resolved in a range of scales at which the velocity field follows the Kolomogorov self-similar structure of turbulence. Hence, we get the possibility to study magnetic induction in a fully developped turbulent flow at low magnetic Prandtl number.

Figure 2 displays isosurfaces of the local induced magnetic energy $\left\langle E_{b}(\mathbf{x}, t)\right\rangle_{T}$ averaged in the time interval $T=[10-410]$, shown at $80 \%$ of its maximum value. For comparison, we also plot isosurfaces of the induced mag- 


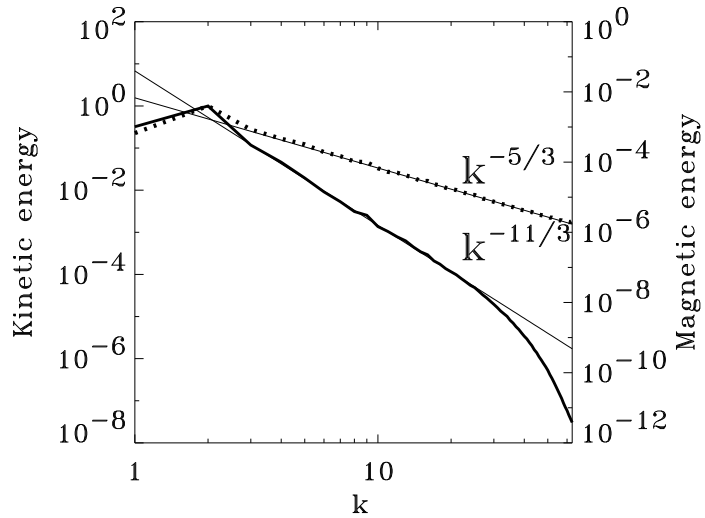

FIG. 1: Magnetic (solid line) and kinetic (dash line) energy spectra computed at $t=210$ for RUN 1 with $\mathbf{B}_{0}=0.1 \hat{\mathbf{x}}$.

netic energy, $\left\langle E_{b, l i n}(\mathbf{x}, t)\right\rangle_{T}$, obtained numerically from a linear approximation based on time averaged velocities: $\lambda \nabla^{2} \mathbf{b}=-\mathbf{B}_{\mathbf{0}} \nabla\langle\mathbf{v}(\mathbf{x}, t)\rangle_{T}$. This is similar to numerical studies based on the averaged flow geometries [7, 19]. When $\mathbf{B}_{0}$ is applied along $\hat{\mathbf{z}}$, in a direction parallel to the rotation axis of the TG eddies, the most intense magnetic energy structures are concentrated round the $z=\pi / 2,3 \pi / 2$ planes, in agreement with the differential rotation of the TG vortex. Moreover, the most intense structures of $\left\langle E_{b}(\mathbf{x}, t)\right\rangle_{T}$ and $\left\langle E_{b, l i n}(\mathbf{x}, t)\right\rangle_{T}$ fields coincide. For $\mathbf{B}_{\mathbf{0}}$ along the $\hat{\mathbf{x}}$-axis, one observes the main induction concentration around the $z=0, \pi$ planes, as expected from a direct inspection from the flow forcing. However, the most intense structures of the $\left\langle E_{b}(\mathbf{x}, t)\right\rangle_{T}$ and $\left\langle E_{b, l i n}(\mathbf{x}, t)\right\rangle_{T}$ fields do not coincide everywhere in that case (see location $(\pi / 2, \pi / 2,0)$ in Fig. 2(bottom), for example). Note also that the linear calculation overestimates the time averaged magnetic fluctuations, whatever the orientation of the applied field. Altogether it shows than one should be cautious when using average velocity fields in the calculation of magnetic induction, particularly if restricted to linear effects. The difference between the fields is probably linked to the large scale electromotive force due to turbulent motions. The influence of this force, as well as the large scale induction topology and its connection with the small scale fluctuations, will be reported in a forthcoming paper [20].

Figure 3 shows the temporal fluctuations of the induced field amplitude, $|\mathbf{b}(\mathbf{x}, t)|$, probed inside the flow at two locations chosen from the previous topological observations, for $\mathbf{B}_{\mathbf{0}}$ along the $\hat{\mathbf{x}}$-axis. This is equivalent to using local probes as in laboratory experiments. The intensity of the induced magnetic field has strong local fluctuations. The point at $(0, \pi, 0)$ is in a region of strong mean induction, whereas the point at $(0.6 \pi, 0.6 \pi, 0.6 \pi)$ is at location of low mean induction (cf. Fig. 2(bottom)). We observe that, occasionally, the induced field gets larger than the applied field. In fact, if small amplitude fluctuations (about 10\%) are induced over time intervals of

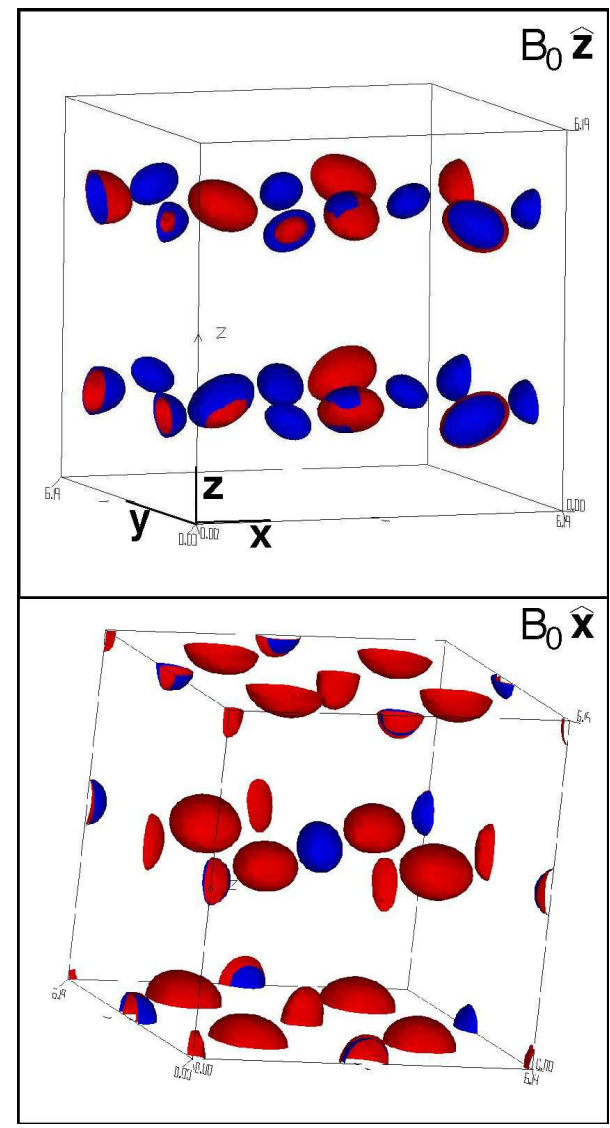

FIG. 2: Topology of the local induced magnetic energy, averaged in time, when $\mathbf{B}_{0}$ is applied along the $\hat{\mathbf{z}}$-axis (top) and along the $\hat{\mathbf{x}}$-axis (bottom) - in red: $\left\langle E_{b}(\mathbf{x}, t)\right\rangle_{T}$; in blue: $\left\langle E_{b, \text { lin }}(\mathbf{x}, t)\right\rangle_{T}-$ (see text). The isosurfaces are plotted at $80 \%$ of the maximum values of the fields : $\max \left\langle E_{b}\right\rangle_{T}=0.0056$ and $\max \left\langle E_{b, \text { lin }}\right\rangle_{T}=0.0063$ for $\mathbf{B}_{\mathbf{0}}=0.1 \hat{\mathbf{z}}$, and $\max \left\langle E_{b}\right\rangle_{T}=$ 0.0041 and $\max \left\langle E_{b, l i n}\right\rangle_{T}=0.0063$ for $\mathbf{B}_{\mathbf{0}}=0.1 \hat{\mathbf{x}}$.

the order of the diffusive time $t_{0}$, much larger variations $(\sim 300 \%)$ can be observed over long time periods, of the order of $10 t_{0}$. These observations are in excellent qual-

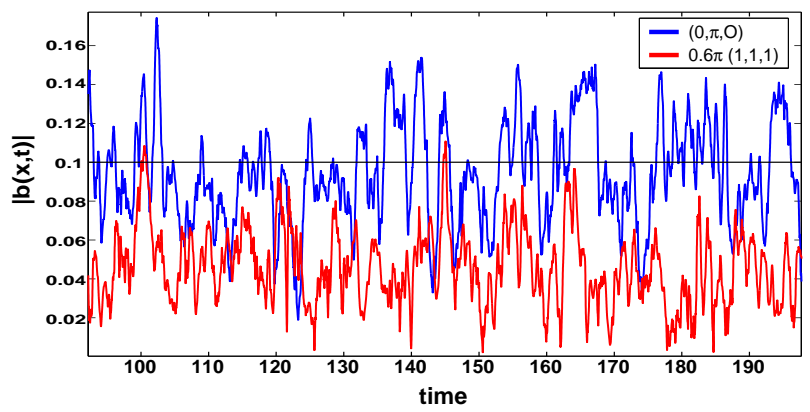

FIG. 3: Time traces of $|\mathbf{b}(\mathbf{x}, t)|$, for $\mathbf{B}_{\mathbf{0}}=0.1 \hat{\mathbf{x}}$, at two fixed points. In blue: $(0, \pi, 0)$, mean value $\langle|\mathbf{b}(\mathbf{x}, t)|\rangle_{T} / B_{0}=$ 0.92 , fluctuation level $|\mathbf{b}(\mathbf{x}, t)|_{r m s} / B_{0}=0.28$. In red: $(0.6 \pi, 0.6 \pi, 0.6 \pi)$ mean value $\langle|\mathbf{b}(\mathbf{x}, t)|\rangle_{T} / B_{0}=0.44$, fluctuation level $|\mathbf{b}(\mathbf{x}, t)|_{r m s} / B_{0}=0.19$. 
itative agreement with the experimental observations at comparable $R_{m}$ and $P_{m}$ [14, 15, 16, 18]. In order to be more quantitative, we analyze the time spectra; we focus on the case with $\mathbf{B}_{0}$ applied along the $\hat{\mathbf{x}}$-axis, but the results are identical when $\mathbf{B}_{0}$ is along $\hat{\mathbf{z}}$. We plot in Figure 4 the power spectra of the temporal fluctuations of the magnetic field component $b_{x}(\mathbf{x}, t)$ recorded at $(0, \pi, 0)$. The higher end of the time spectrum follows a behavior close to $f^{-11 / 3}$, as can be expected from the spatial spectrum using the Taylor hypothesis of "frozen" field lines advected by the mean flow [18]. In addition, for frequencies roughly between $1 / t_{0}$ and $1 / 10 t_{0}$, the time spectrum develops a $1 / f$ behavior, as observed in experimental measurements [15]. It is not present on the spatial spectrum in Figure 1, and thus appears as a distinctive feature of the time dynamics of the induced field. It is also independant of dynamo action, as it is also observed in the Karlsruhe experiments [16]. Finally, our numerical
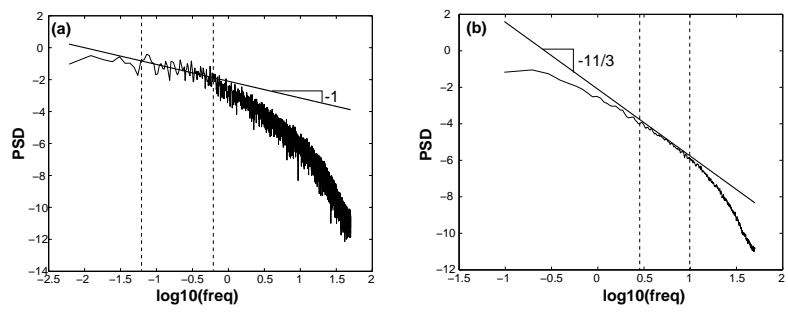

FIG. 4: Power spectral density of the magnetic field fluctuations of $b_{x}(\mathbf{x}, t)$ in time, recorded at space location $(0, \pi, 0)$, when $\mathbf{B}_{\mathbf{0}}=0.1 \hat{\mathbf{x}}$. (a) PSD computed as averages over Fourier transforms calculated over long time intervals $\left(\sim 164 t_{0}\right)$ to emphasize the low frequency behavior; (b) PSD estimated from Fourier transforms over shorter time intervals $\left(\sim 10 t_{0}\right)$. The behavior is identical for the $b_{y}(\mathbf{x}, t)$ and $b_{x}(\mathbf{x}, t)$ field components.

study reveals one remarkable feature: the $1 / f$ behavior is a global feature. It is observed on the fluctuations of the magnetic energy, as shown in Figure 5 (as a $f^{-2}$ scaling regime). We thus propose that it results from induction processes which have contributions up to the largest scale in the system.

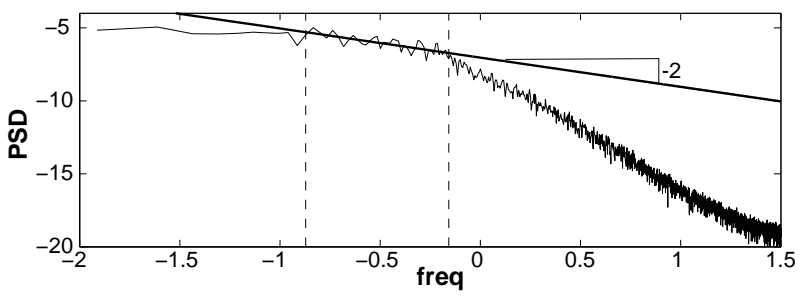

FIG. 5: Power spectral density of the time fluctuations of the magnetic energy $E_{b}(t)=\left\langle\mathbf{b}^{2}(t)\right\rangle / 2$, intergrated over space.

To summarize, the mixed numerical scheme proposed here proves to be a valuable tool for the study of magnetohydrodynamics at low magnetic Prandtl numbers.
We have considered here the response to an externally applied field. The time behavior of magnetic field fluctuations is found in excellent agreement with experimental measurements. It has also revealed that the $1 / f$ regime detected locally traces back to the global dynamics of the flow. Future work will analyze the contribution of turbulent fluctuations to the large scale magnetic field dynamics, and the influence of the magnetic Prandtl number on the threshold of the dynamo instability.

Acknowledgements: We thank J.-P. Bertoglio, P. Odier and A. Pouquet for fruitful discussions. This work is supported by CNRS ATIP/SPI, PCMI and GdRDynamo. Computations performed on an Alineos PC cluster (OCA) and at IDRIS.

[1] R. Stieglitz, U. Müller , Phys. Fluids, 13, 561, (2001)

[2] A. Gailitis et al., Phys. Rev. Lett., 86, 3024 (2001).

[3] Special issue, MHD dynamo experiments, Magnetohydrodynamics, 38(1-2) (2002).

[4] R. Kaiser, A. Tilgner, Phys. Rev E, 60, 2949 (1999).

[5] F. Stefani, G. Gerberth, A. Galaitis, Numerical simulations for the Riga dynamo, in Laboratoty Experiments on Dynamo Action, Riga, Latvia, 14-16 June 1998, edited by O. Lielausis, A. Galaitis, G. Gerberth and F. Stefani.

[6] N.L. Dudley and R.W. James, Proc. R. Soc. London, Ser. A 452, 407 (1989).

[7] L. Marié, J. Burguete, F. Daviaud, J. Léorat, Eur. Phys. J. B, 33, 469 (2003).

[8] M. Meneguzzi, U. Frisch, A. Pouquet, Pys. Rev. Lett., 47, 1060 (1981).

[9] C. Nore, M. Brachet, H. Politano, A. Pouquet, Phys. Plasmas, 4,1 (1997).

[10] C. Nore, M.-E. Brachet, H. Politano, A. Pouquet, "Dynamo action in a forced Taylor-Green vortex", 51-58, in Dynamo and Dynamics, a mathematical challenge. Nato Science Series II, Vol. 26, edited by P. Chossat, D. Armbruster and I. Oprea (Kluwer Academic, Dordrecht), (2001).

[11] A.A. Schekochihin et al., arXiv astro-ph/0308336 (2003).

[12] G.A. Glatzmaier and P.H. Roberts, Nature, 377, 203 (1995).

[13] J.-P. Chollet and M. Lesieur, J. Atmos. Sci., 38, 2747 (1981)

[14] N.L. Peffley, A.B. Cawthrone, D.P. Lathrop, Phys. Rev. E, 5287 (2000).

[15] M. Bourgoin et al. Phys. Fluids, 14, 3046 (2001).

[16] R. Stieglietz, U. Müller, The Karsruhe dynamo experiment, Wissenschaftliche Berichte, FZKA report No.6756 (2002).

[17] H.K. Moffatt, J. Fluid Mech., 11, 625 (1961).

[18] P. Odier, J.-F. Pinton, S. Fauve, Phys. Rev. E, 58, 7397 (1998).

[19] M. Bourgoin, P. Odier, J.-F. Pinton, Y. Ricard, Phys. Fluids, preprint, (2003).

[20] Y. Ponty et al., Turbulent fluctuations and large scale magnetic fields, to be submitted (2004). 